\title{
Civilisations
}

Revue internationale d'anthropologie et de sciences

humaines

44 | 1997

Les peuples des forêts tropicales

\section{L'Indien, le Noir et le CabocloAmazonie indigène ou Amazonies métisses?}

Didier de Laveleye

\section{(Q) OpenEdition \\ Journals}

Édition électronique

URL : https://journals.openedition.org/civilisations/1625

DOI : 10.4000/civilisations. 1625

ISSN : 2032-0442

\section{Éditeur}

Institut de sociologie de l'Université Libre de Bruxelles

\section{Édition imprimée}

Date de publication : 1 janvier 1997

Pagination : 152-167

ISBN : 2-87263-122-4

ISSN : 0009-8140

Référence électronique

Didier de Laveleye, «L'Indien, le Noir et le CabocloAmazonie indigène ou Amazonies métisses ? », Civilisations [En ligne], 44 | 1997, mis en ligne le 29 juin 2009, consulté le 21 septembre 2021. URL: http://journals.openedition.org/civilisations/1625; DOI : https://doi.org/10.4000/civilisations. 1625 


\section{- L L INDIEN, LE NOR ET LE CABOCLO: Amazonie indigène ou Amazonies métisses?}

Didier de LAVELEYE 


\section{HISTOARES AMÉPNMDENENES}

Ne nous y trompons pas, l'amazonie n'est plus - et n'a peut-être jamais été - une impénétrable forêt peuplée ça et là par quelques petites bandes d'Amérindiens, derniers vestiges d'un archaïsme précolombien. Trop souvent on tente de nous vendre cette image filtrée d'exotisme, galvaudée à l'excès par nos phantasmes les plus douteux sur la sauvagerie. Cette production médiatique, voire même (pseudo-) scientifique, court le risque dentaner plus encore notre réelle capacité à saisir les véritables enjeux environnementaux, sociaux et culturels qui traversent l'Amazonie contemporaine.

Léquation qui fonde cette représentation simpliste du monde amazonien se base sur l'idée que la forêt aurait formé une barrière naturelle entre notre civilisation destructrice et les premiers habitants de l'Amérique. Certes, il est vrai que l'Amazonie a quelquefois servi de refuge aux indigènes fuyant l'oppression coloniale, comme le feront plus tard de nombreux esclaves africains. Mais rapidement, des nilliers de petits colons européens, ne trouvant pas leur compte dans les villes marchandes ni auprès des grands exploitants miniers ou agricoles, suivront le même chemin de «la liberté» en s'enfonçant à leur tour dans ce milieu tropical humide. Aujourd hui encore l'Amazonie représente pour des millions de pionniers, cette même voie vers l'autonomie, une terre à soi, un avenir meilleur. Dans cette perspective on comprendra facilement que cet espace n'a jamais vraiment été celui d'une frontière entre le monde amérindien et occidental car, outre son précoce attrait économique (bois, latex, cacao, etc), il fut aussi plus largement celui de la rencontre entre tous les exchus du système colonial dominant.

Des études ethno-archéologiques prouvent aujourd'hui qu'à l'époque précolombienne, contrairement à ce que nous croyions, l'Amazonie était peuplée par de grands ensembles politiques, pouvant regrouper le long des fleuves un étonnant tissu urbain, d'où partaient même des routes bordées d'arbres fruitiers [Lévi-Strauss, 1994; Roosevelt, 1993\}. Des traces de terrassements tendent aussi à démontrer que leur système économique et agricole devait être beaucoup plus érendu qu'il ne l'est actuellement. Cette irruption d'une importante dimension historique en Amazonie, outre le fait qu'elle suffit à démonter l'image simpliste que nous dénoncions plus haut, nous éclaire davantage sur l'impact, même indirect, qu'a dû provoquer l'arrivée des colons européens sur le Continent. Bien que plusieurs hypothèses demeurent possibles quant à la raison de cette dislocation rapide des systèmes sociaux complexes, il est indéniable que cette rupture instaura une discontinuité dans l'histoire des sociétés amérindiennes, modifiant tout à la fois leur relation au temps, leurs organisations politiques, leurs rapports économiques, et sans doute bien davantage encore.

En conséquence, comme le trote claude léviStrauss, si les sociétés amérindiennes ont pu être considérées comme étant historiquement "froides", c'est que la manière subjective dont elles conçoivent leur devenir se caractérise par la volonté de neutraliser le changement, mais «dans l'espoir certes vain de se mettre à l'abri, et ne sachant même plus combien elles ont changé, elles se cramponnent à leur condition présente et la pro- 
clament toujours telle que les dieux ou les ancêtres l'instaurèrent» [Lévi-Strauss, 1993: 9]. Or, cette stratégie sociologique n'a certainement pas pu être adoptée par l'ensemble du monde amazonien. Suite, probablement, aux fissions d'importants espaces sociaux précolombiens, relayées par une criante chute démographique qui mena beaucoup de communautés à la disparition pure et simple, de nombreux groupes ont préféré adopter une stratégie inverse en acceptant -ou ne pouvant plus refuser- une modification plus ou moins profonde de leur organisation sociale et culturelle. Dans les cas extrêmes, l'atomisation brutale des populations amérindiennes provoqua souvent un démembrement irréversible de leurs unités sociales, conduisant même la plupart d'entre elles à perdre, au fil des générations, le sentiment - et parfois le souvenir - d'avoir été l'Indien de telle ou de telle ethnie. D'autres par contre adoptèrent une véritable stratégie adaptatrice en fusionnant avec

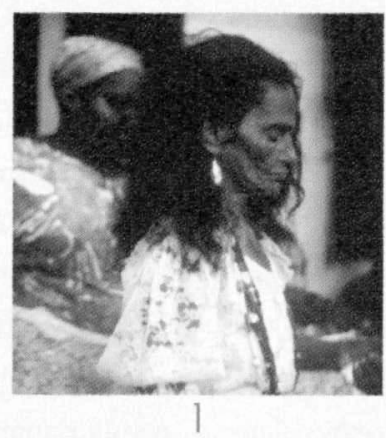
des groupes également affaiblis démographiquement, même si ceux-ci pouvaient être traditionnellement ennemis ou parler des langues différentes!

Claude Lévi-Strauss lui-même rapporta un événement analogue lors de l'expédition ethnographique qu'il effectua au Brésil en 1937. Il avait rencontré un groupe d'Indiens Nambikwara qui, suite à une importante épidémie de grippe, décida d'adopter in extremis une stratégie fusionnelle avec une bande «ennemie» qui parlait un autre dialecte, inconnu de l'ethnologue. Dans ce cas précis, l'opération consista à déclarer que tous les hommes adultes devaient désigner les femmes de l'une et l'autre bande par le terme "sœur" et, inversement celles-ci devaient appeler «frère» les hommes du groupe voisin. De par le système matrimonial indigène, ce plan plaça tous les enfants dans la position d'époux potentiels des enfants de l'autre ethnie, «si bien que, par le jeu des intermariages, les deux bandes auraient fusionné dès la prochaine génération» [LéviStrauss, 1955: 362].

Ce type de manipulation du système social démontre parfaitement que toutes ces sociétés, aux moments les plus critiques de leur histoire, n’hésitent pas à précipiter les effets qui perturbent excessivement leur tradition, à innover. Malheureusement, rares sont encore les études qui se sont sérieusement penchées sur ce phénomène de «réchauffement» de l'histoire des sociétés postcolombiennes en réunissant à la fois du matériel ethnographique, archéologique et ethno-historique ${ }^{[1]}$. Il n'y a pas de doute cependant que l'ensemble du monde amazonien a dû ressentir -certes, à des degrés divers- l'effet de ces processus qui ont dû se précipiter dès la déstructuration des grands ensembles politiques. De cette manière, combien de communautés amérindiennes n'ont pas pris garde aux risques de perdre leur identité ancestrale pour se retrouver finalement dans la masse insaisissable des métis? Car, si les pratiques fusionnelles entre Indiens ne sont peut-être plus toujours vraiment visibles aujourd'hui, celles entre Indiens et Noirs, entre Indiens et Blancs, ou encore entre Indiens et Métis 
se sont, à l'évidence, étendues à l'ensemble du bassin amazonien. Aujourd'hui, cette frange de l'Amazonie que l'on traite généralement de détribalisée et d'acculturée, ou plus spécifiquement de Caboclo au Brésil, représente au moins plus des deux tiers de sa population. C'est elle aussi qui exprime sans doute le plus brutalement ce qu'on peut appeler le versant "chaud» de l'histoire amérindienne...

On aurait pourtant tort d'accentuer la fracture entre ceux que l'on considère encore conme les «vrais» Indiens et les «Autres». Ne commettrions-nous pas là un des plus grands cynismes de notre histoire coloniale en reconnaissant des droits aux uns tout en niant l'existence sociale de ceux que nous avons emportés avec nous dans la tourmente "civilisatrice»? Ne voir qu'une irréductibilité entre ces deux sociétés c'est aussi s'interdire d'analyser et de comprendre le mécanisme qui les distingue. Car, toute tentative d'établissement d'un modèle nertinent de l'espace social et culturel amazonien passe par la prise en compte de sa dimension historique, allant de la «rupture» colonisatrice à la reformulation des systèmes sociaux et des identités ethniques.

\section{AMAZONE CABCCLLA, AMAZZOMES MÉTISSES}

Evidemment chaque communauté amazonienne relève aujourd hui d'une histoire particulière qui n'est réductible à aucune autre. Cependant nous avons vu que celle-ci était sans doute caractérisée par quelques grandes tendances, comme, par exemple, la cristallisation des traditions ancestrales (société à «histoire froide"), l'atomisation en groupuscules (fissions) pouvant aller jusqu'à l'isolement de familles restreintes, ou encore la fusion inter-ethnique. Il va de soi également que chacune de ces situations peut présenter d'importantes variations sans pour autant être exclusives l'une de l'autre. Ainsi, un groupe qui s'est disloqué pour un moment peut très bien avoir refusionné par la suite et figer finalement son organisation interne. Inversement, la fusion de deux communautés peut se solder par l'éclatement radical en petites unités conjugales. En fait, cette souplesse historique est déjà traditionnellement caractéristique de nombreuses sociétés amazoniennes qui, en raison de leur système politique, connaissent de véritables cycles centrifuge/centripète, de telle sorte que l'unité villageoise relève bien souvent de l'association momentanée ${ }^{(2)}$. Cependant, ce qui va nous intéresser ici c'est quand cette dynamique a obtenu une telle extension qu'elle dépasse la sphère endo-ethnique au point que l'identité du groupe s'en trouve transfigurée. Un premier exemple nous avait été donné par la fusion de deux ethnies amérindiennes. Au Brésil, la situation la plus répandue (et largement majoritaire) est celle des Caboclo, issus de profondes déstructurations ethniques, et vivant aujourd'hui, dans la plupart des cas, en petites familles restreintes, disséminées sur tout le bassin amazonien [3].

le caractère extrênement polysémique du terme Caboclo démontre déjà la difficulté - tant du côté de la société brésilienne que de celui des sociologues - à s'entendre sur le sens à donner à I'histoire dont ces hommes sont le produit. Sans nous étendre sur les détails de ses multiples usages et significations cette appellation générique renvoie souvent à l'image d'un Indien acculturé ou métis et, ce qui est plus significatif 
encore, démuni d'une origine ethnique précise. L'étymologie la plus pertinente de ce terme relève cependant d'une origine linguistique tupi qui signifierait tout simplement «qui vient de la forêt» [Grenand \& Grenand, 1990]. C'est également le sens qu'on lui donne dans les multiples cultes syncrétiques afro-amérindiens du pays où les esprits de Caboclo sont effectivement ceux qui viennent de la forêt. Ils représentent cette figure emblématique de l'homme amazonien, hésitant quelquefois sur leur origine amérindienne (parfois nommée), européenne ou africaine; et préfèrent souvent se présenter comme de simples paysans métis. Le champ sémantique de cet homme de la forêt, pris à la fois dans son espace social et religieux, dévoile donc essentiellement une nature post-colombienne - coloniale - plutôt qu'une référence amérindienne explicite. Ainsi, ceux qui se désignent (ou que l'on désigne) par le terme Caboclo articulent sur-

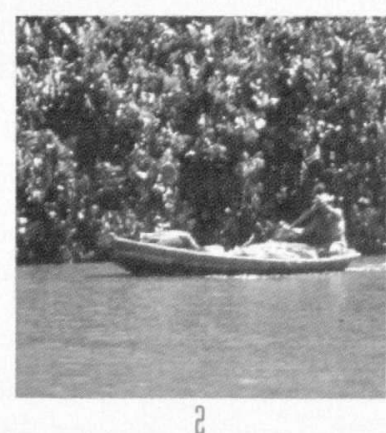
tout leur existence dans leur espace social contemporain, que nous désignerons ici par néoamazonien.

Il convient donc de relativiser toute définition qui enfermerait cette catégorie de la population amazonienne dans un cadre trop étroit. Les Caboclo ne forment pas un ensemble culturel cohérent -une ethnie- car si globalement ce sont peut-être les mêmes mécanismes historiques qui ont conduit à leur formation (stratégies fusionnelles et fissionnelles), celles-ci se sont déroulées (et opèrent encore) dans des contextes socio-culturels souvent fort dissemblables.
Prenons par exemple, pour clarifier cette polysémie, le cas des actuels Tikuna. Cette population amérindienne s'autodésignait comme Caboclo jusqu'à une période récente [Pacheco de Oliveira, 1990], tendant par là à ne plus se distinguer des autres petits colons métis qui, comme eux, récoltaient du latex pour le compte d'un patron. La déstructuration progressive de l'organisation sociale indigène depuis le XVII ${ }^{2}$ siècle, en même temps que son adaptation - souvent forcée - aux contraintes de la société coloniale marchande, pouvaient présager une uniformisation généralisée de cette population forestière blanche et amérindienne. Cependant, à partir des années '70, des événements politiques liés à des questions territoriales, l'ouverture d'une route (la Perimetral Norte) et l'arrivée de nouveaux exploitants forestiers, ont provoqué la résurgence d'une identité ethnique et politique tikuna. Cette population caboclo s'est alors dédoublée en rétablissant une limite - devenue quelque peu subjective - entre Indiens et non Indiens. Ainsi, ceux qui continuèrent à s'identifier comme Caboclo furent rejetés du côté des nouveaux migrants -donc du monde des Blancs-et, de ce fait, entrèrent également en conflit avec ceux qui se réapproprièrent une indentité amérindienne spécifique ${ }^{[4]}$. Situation d'autant plus paradoxale que si l'origine de ces conflits concernait l'intégrité d'un territoire et l'accès à ses ressources, c'est l'ensemble de la population rurale qui subissait ces nouvelles pressions de la part des nouveaux exploitants forestiers. 
Le cas Tikuna démontre parfaitement que si l'appellation Caboclo peut désigner un métissage et même souvent un remaniement de la référence ancestrale, il ne signifie pas pour autant que les anciennes origines ethniques soient systématiquement effacées. Cependant, dans la plupart des situations similaires où un front pionnier récent s'est installé sur des terres déjà occupées depuis plusieurs générations par des Caboclo, ces derniers n'y ont pas répondu pour autant par une recristallisation de leurs identités ethniques. Le caractère largement inarticulé de cette population démontre davantage sa difficulté à se forger une identité singulière, c'est-à-dire métisse.

Officiellement, les multiples conflits territoriaux qui enveniment l'Amazonie depuis la construction de la Transamazonienne et son afflux de pionniers, résultent de la contradiction entre deux légitimités: d'une part, l'obligation pour l'État de garantir l'intégrité des

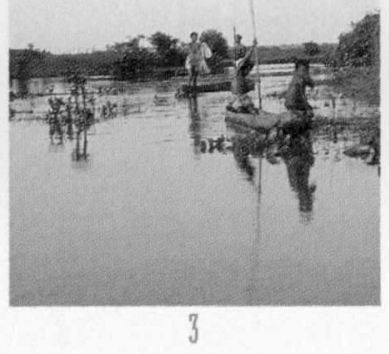

«reconnus» et non pas simplement octroyés par l'État, tandis que leurs droits territoriaux sont déclarés «originaires», c'est-à-dire antérieurs à l'État brésilien [...] : ce sont proprement des droits historiques " [Carneiro da Cunha, 1990: 13]. Durant les débats parlementaires, on proposa de limiter ce privilège statutaire aux Indiens nonacculturés; proposition qui fut retirée de justesse par l'Assemblée constituante, mais que l'on retrouva sous forme d'un décret gouvernemental établissant des procédures différentes pour la reconnaissance légale des droits territoriaux des «Indiens acculturés» et des «Indiens non-acculturés». Cette intrusion d'un concept sociologique - l'acculturation - dans une réglementation juridique démontre parfaitement l'aspect pernicieux de ce projet politique (sur lequel se sont entendus les deux parties) qui consiste à scinder l'Amazonie en deux espaces contradictoires : les territoires «de la tradition» et les territoires indigènes et, de l'autre, son besoin d'offrir au pays un développement économique. Ces dernières années la scène politique brésilienne s'est largement cristallisée sur ces deux exigences - nationaliste et indigéniste -, en accentuant le fossé (somme toute fort idéologique) entre populations indigènes et allogènes. L'importante victoire remportée par les organisations indigénistes au cours de l'élaboration de la nouvelle Constitution brésilienne de 1988 n'a fait qu'exacerber cette dualisation excessive de l'Amazonie. Comme le souligne Manuel Carneiro, les droits des populations indigènes sont, depuis, espaces de développement national. Pour être reconnu en tant que minorité et surtout pour espérer préserver son intégrité sociale, culturelle et territoriale, il faut aujourd'hui appartenir à un seul modèle de «tradition», figé dans l'histoire pré-colombienne. Dans ce contexte, les communautés caboclo (ou «acculturées») ne bénéficient d'aucune protection juridique, de telle sorte qu'elles sont tiraillées entre leurs influences indigènes et nationales (blanches) sans jamais pouvoir prétendre à une spécificité sociale ou culturelle. Soit elles sont identifiées comme population indigène et dans ce cas seulement elles ont le droit 
de tenter une reconnaissance légale de leurs terres, soit elles sont refoulées du côté de la masse des nouveaux pionniers (ce qui est largement plus fréquent) et n'ont, à ce titre, pas plus de droit que l'ensemble des nouveaux migrants venus des grandes villes du sud.

Les quelques études portant sur les Caboclo amazoniens s'entendent pourtant pour considérer que ceux-ci doivent être culturellement, sociologiquement et économiquement distingués de l'ensemble des fronts pionniers qui accompagnent les pôles de développement économique de l'Amazonie. Les Caboclo, comme les communautés indigènes, se distinguent du reste de l'espace socio-économique brésilien dans la mesure où leur spécificité sociale et culturelle s'incrit dans un environnement naturel englobant simultanément leurs rapports pratique et symbolique. Leur espace ne se limite pas à son aspect économique, mais est aussi un espace

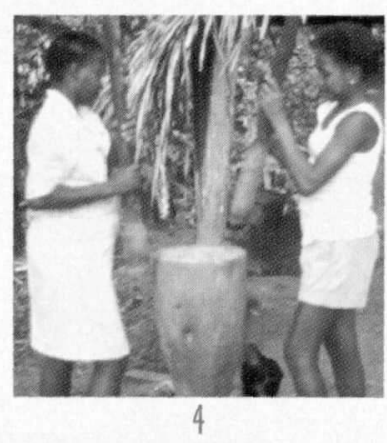

péennes) un corpus original de savoirs et de techniques, leur permettant d'élaborer un modèle optimal de gestion et d'exploitation du milieu amazonien ${ }^{[5]}$. Cette querelle, aussi absurde soitelle, n'est parvenue à ce jour qu'à alimenter la désinformation qui envenime l'Amazonie contemporaine, en ne lui reconnaissant pas une pluralité où cohabitent traditions, modernité et métissage culturel. Si, effectivement, les communautés caboclo partagent avec les Amérindiens certaines connaissances et techniques qui orientent leur gestion de l'environnement sur des pratiques globalement non destructrices (comme l'agriculture itinérante sur brûlis complétée par de socialisation des forces naturelles, gérées à partir d'une connaissance particulière de l'environnement où se mêlent les exigences matérielles et les représentations religieuses [Galvão, 1976].

Certains se sont plu à considérer que le Caboclo représenterait une forme amoindrie de culture amérindienne, enlisée dans une dépendance économique vis-à-vis du monde extérieur et, plus particulièrement, englobée dans l'économie de marché. D'autres anthropologues ont tenu le discours inverse, en argumentant le fait que les Caboclo seraient parvenus à extraire des cultures indigènes et allogènes (essentiellement euro- des produits de cueillette et de chasse), cet «acquis» culturel reste largement vulnérable. Surtout, la spécificité caboclo basée sur un ajustement entre deux modèles de société, reste largement tributaire de l'environnement politique (pour ne pas dire idéologique) qui refuse jusqu'à présent de reconnaitre tout simplement son droit à l'existence.

On croit souvent - à tort - que prendre parti pour la défense des Amérindiens c'est automatiquement protéger le milieu écologique qui les abrite. Telle attitude, sans nuance, si elle permet effectivement de renforcer quelques pôles de «tradition» et d'écologie, participe également à déstabiliser l'ensemble du monde néo-amazonien. Il n'est pas rare aujourd'hui que l'on demande à l'opinion publique internationale de cautionner l'expulsion de populations caboclos d'un territoire déclaré «indigène». Ce sont exactement les 
mêmes genres de pratiques - la non reconnaissance d'un droit - qui ont conduit, depuis un demi millénaire, des millions d'Amérindiens à perdre l'armature sociale et culturelle (et environnementale) qui guidait leurs savoirs et pratiques du milieu, pour les mettre au service d'un exploitant forestier, d'un chantier hydroélectrique, d'un exportateur de viandes et de peaux exotiques... Renforcer les droits des uns au détriment des droits fondamentaux des autres, c'est à mon sens prendre parti pour une cause dont les conséquences s'avèreront non seulement inacceptables mais également dangereuses. Ainsi, si nous insistons ici sur le rôle crucial - central même, vu son importance démographique - que peut jouer le Caboclo en Amazonie, c'est moins pour glorifier son métissage que pour insister sur le danger qui consiste à nier son existence en ne lui donnant pas les moyens de la consolider et en le refoulant du côté de la culture nationale et de son modèle de développement économique. Ce "projet» politique, ce "manichéisme» amazonien, ne peut que conduire à renforcer les stratégies prédatrices tout en fragilisant les sociétés amérindiennes que l'on croit préserver en accentuant de plus en plus leur confrontation avec la société nationale. Il est grand temps de reconnaître à l'Amazonie son caractère multiple (et non simplement dual) en cherchant dans ses zones tampons, ses cultures «mixtes», une voie durable tant pour la forêt que pour ses habitants.

\section{LA RENCONTHE OU NOHA ET OE L''WDIECH}

Lopposition Blanc-Indien, outre le fait qu'elle occulte l'existence d'une large population métisse en Amazonie, contribue également à sous-estimer la présence du Noir au sein du monde rural brésilien. Ici encore, à l'inverse du discours idéologique dominant, la règle est bien celle de la rencontre, des mélanges culturels et des multiples syncrétismes.

La traite africaine transplanta vers le Brésil plusieurs millions d'esclaves dans le but avoué de les substituer à la défaillante servilité des Indiens ${ }^{[b]}$. C'est d'abord dans les grandes exploitations agricoles -de coton, de canne à sucre, de cacao et de café- que ces hommes et femmes furent envoyés en masse dès le XVIème siècle. Face à la dureté du régime esclavagiste et de son système répressif, nombreux furent ceux qui tentèrent de fuir en s'enfonçant dans la forêt toute proche. On estime que chaque fazenda (ferme coloniale) comptait ainsi son contingent d'esclaves fugitifs, retranché en communauté autonome et installé en des lieux difficiles d'accès afin d'échapper aux fréquentes représailles de l'armée coloniale. Ce phénomène pu prendre de telles proportions que certaines de ces communautés - ces quilombos - étaient composées de plusieurs milliers d'esclaves et pratiquaient même, dans une semi-clandestinité, des relations commerciales avec les colons. Le quilombo de Palmares fut sans aucun doute le plus prestigieux puisque celui-ci s'était érigé en un véritable royaume armé qui brava l'ordre esclavagiste pendant plus d'un siècle (1570-1694). Mais d'autres quilombos, situés en plein cneur de l'Amazonie, sont parvenus à maintenir leur intégrité et réapparaissent aujourd' hui, comme nous le verrons plus bas, sous la forme d'un enjeu politique qui rappelle à plusieurs égards celui des communautés amérindiennes. 
Si la traite ne subsiste plus aujourd'hui que dans la mémoire des Afro-Brésiliens, à ce titre cette époque marque un temps fondateur de toute une culture populaire, elle est présente à travers tout le Brésil jusqu'au plus profond de l'Amazonie. Cet espace multi-ethnique, appréhendé à travers son histoire, révèle une dimension curieusement ignorée - presque occulte -, celle de la rencontre entre le Noir et l'Indien, et pourtant aisément visible aujourd'hui, tant dans les activités folkloriques que religieuses. À ce titre le Maranhão est sans aucun doute la province amazonienne la plus riche en enseignement. Située à l'extrême est du Bassin de l'Amazone, à cheval entre la forêt tropicale et les plaines arides du Nordeste brésilien, cette région fut marquée dès le XVIIème siècle par une forte implantation esclavagiste tout en abritant de nombreux groupes d'Indiens (essentiellement Tupi). Or, au cœur de cette «mosäque» de cultures, d'étonnantes traditions folkloriques et religieuses

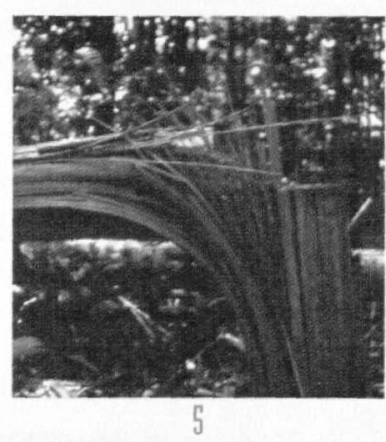

Sans entrer dans le détail de chacune de ces activités $^{[7]}$, signalons simplement qu'à la fois les acteurs et surtout les rôles que ceux-ci jouent lors de ces célébrations, renvoient systématiquement à des rapports inter-ethniques entre lesquels le métissage apparait parfois comme un fait, ni stigmatisé ni glorifié, permettant de reconstruire le lien nécessaire entre passé et présent. Dans les communautés de culte Mina, par exemple, nous avons noté l'existence d'un mythe fondateur de la population métisse afro-amérindienne du Maranhão. Ce fabuleux récit relate la rencontre entre deux lignées d'ancêtres (mythiques) : un roi africain, suite à une traversée en mer qui se solda perpétuent inlassablement une vaste épopée-celle de leurs rencontres, leurs affrontements ou leurs alliances- où s'entrecroisent héros, saints, esprits, guerriers ou simplement paysans; tantôt Indiens, Noirs ou Blancs, ou mieux encore Caboclo. Cette célébration du métissage est donnée à voir en plusieurs actes: dans la rue au moment des fêtes folkloriques Bumba-Meu-Boi, à la maison parfois lors des danses Tambor de Crioula, à l'église aussi pendant les cérémonies catholiques du Divinio (Fête-Dieu), et surtout dans les temples des communautés afro-brésiliennes du culte Mina, au cours des grands rituels de possession. par une «trahison» portugaise (symbolisant ici la traite), s'égare en forêt. Il est alors accueilli dans le village du «Roi des Indiens» qui avait fui un massacre ayant décimé tout son peuple ${ }^{[8]}$. L'épopée du métissage afro-amérindien exprime donc dans ce mythe le contexte historique de leur union : la traite des Noirs et le massacre des Indiens. Or, ce qui est symptomatique ici c'est que cette alliance s'effectue dans le récit afro-amérindien exactement de la même manière que lors de l'événement - historique cette fois relevé par Claude Lévi-Strauss à propos de deux bandes indiennes. En effet, la même stratégie parentale-l'adoption des enfants de l'un et l'autre groupe - y est décrite afin de permettre leur fusion inter-ethnique et donc de justifier leur métissage.

Le culte Mina, bien qu'il soit à l'origine un phénomène religieux propre aux esclaves du Maranhão, s'est répandu à travers tout le Nord du 
Brésil, puisqu'on le retrouve également dans les États du Pará et de l'Amazonas. Aussi cette religion connaît d'importantes variations en fonction des lieux et des personnes qui la pratiquent. Dans les zones rurales elle s'est particulièrement étoffée en intégrant - et fusionnant - à la fois ses origines noires et amérindiennes. Cependant, de la même manière que leur métissage semble être un fait de conscience justifié et parfois réglementé par un "plan», leur alliance culturelle et religieuse passe par une structuration précise des identités qui la composent. Ainsi, tout en accueillant l'ensemble des divinités et des esprits propres aux mythologies afro-brésiliennes et amérindiennes, la religion Mina distingue trois panthéons auxquels se réferrent trois cultes orientés sur un symbolisme des origines «ethniques» du peuple afro-amérindien ${ }^{[9]}$ : le panthéon Agua Salgada (l'eau salée), établit une médiation avec l'origine sacralisée (la traversée en

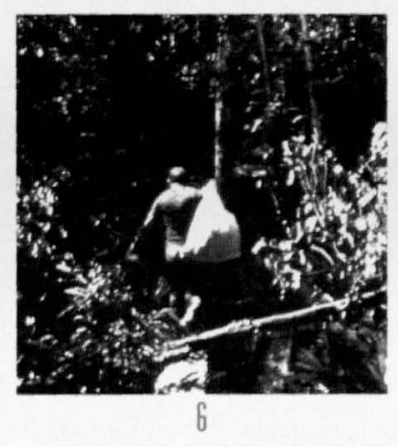
d'agriculture et d'élevage. On constate donc ici qu'en offrant aux fidèles un système unifié de communication avec des esprits et divinités liés à une ancestralité africaine, européenne, amérindienne et caboclo, le culte Mina exprime concrètement la complexité ethno-sociologique de l'Amazonie contemporaine. Cette religion permet à la fois d'exprimer un particularisme ethnique - réel ou imaginaire peu importe ici - tout en englobant l'ensemble de la population - allogène et indigène - dans un espace naturel, social, culturel, religieux et historique commun.

L'épopée du métissage nous apparait, à la lumière de son système symbolique, comme un mer) de l'esclave et, à ce titre, se compose exclusivement de divinités allogènes (principalement africaines, mais aussi européennes); l'Agua Doce (l'eau douce), par contre, plonge dans le fond des rivières du pays qui, comme dans le chamanisme amérindien, abritent une multitude d'esprits de la nature (dauphins, serpents, oiseaux,...) mais aussi des esprits d'anciens chamans (pajé), figures mystérieuses et puissantes de l'Indien; et enfin, la Mata (la forêt) se tourne vers l'espace emblématique du Caboclo, vers son histoire coloniale, faite de métissages, de conflits, de fuites, mais aussi plus simplement de chasse, de pêche, fait social dont la portée dépasse la simple idiosyncrasie des AfroBrésiliens. Au contraire, elle se laisse lire non seulement comme une ethno-histoire, mais aussi comme une praxis de l'identité multi-ethnique néoamazonienne. En ce sens, si dans l'espace urbain de São Luís, capitale du Maranhão, ce sont les divinités africaines de l'Agua Salgada - les Orixas - qui prédominent, dans les zones rurales ce sont au contraire les esprits de la forêt et des rivières qui investissent surtout l'espace rituel. Cependant, audelà de ces particularismes, le côtoiement de ces trois panthéons - l'allogène, l'indigène et le cabolco - opère partout, de telle sorte que c'est à travers le jeu de leur rencontre que s'expriment le mieux les caractéristiques de chacun. C'est dans cet ordre d'idées que nous avons rencontré un Caboclo qui, lors d'un culte Mina, s'est laissé posséder par un esprit de caboclo (appartenant au 
panthéon de la Mata) avant d'entreprendre un rituel thérapeutique avec des esprits appartenant à l'univers symbolique amérindien (de l'Agua Doce). La lutte symbolique que ce guérisseur joua devant l'assistance en vue de soigner une femme atteinte d'infécondité s'accomplit donc sur deux registres : tout d'abord l'homme se transforma, via la transe de possession, en une représentation divinisée de son identité caboclo pour ensuite, sous cette puissante forme, passer dans le monde fantastique de la surnature amérindienne et y combattre, dans la pure tradition chamanique, des esprits pathogènes responsables des troubles de sa patiente. En ce sens, tout se passa comme si le guérisseur caboclo affirma préalablement son identité caboclo avant d'entreprendre un rite (la lutte chamanique) où il aurait pu être confondu -à tort- avec un Amérindien. De cette manière les hommes «de la forêt» rejouent symboliquement et physiquement (via la transe) la fusion culturelle dont ils sont le produit. Ce système nous présente également une limite à l'autochtonie du C'aboclo: réelle en ce qui concerne son appropriation de l'espace et des techniques qu'il met en ouvre (en l'occurrence amérindienne), mais "différente» en ce qui concerne son identité d'homme amazonien.

\section{LE QUULOMAO OU LE MYTHE Q'UNE TEARE AFAICANE RETROUUÉE}

L'espace néo-amazonien se définit donc, comme nous venons de le suggérer, à la fois par ses multiples origines culturelles indigènes et allogènes, tout en se ménageant - timidement peutêtre - une singularité. Cependant, il convient d'insister sur le fait que, quelle que soit la forme que ces cultures «mixtes» peuvent prendre dans leur environnement multi-ethnique, celles-ci se fondent dans et avec un espace naturel et culturel; en ce sens elles sont aussi autochtones.

Ainsi, les fameux quilombos (les communautés d'esclaves fugitifs ou marrons) sont sans doute le résultat des conditions historiques qui ont conduit à leur apparition, mais leur forme actuelle s'est additionnée, au fil des générations, de relations spécifiques avec l'espace - naturel et social - qu'ils occupent depuis leur formation. Autrement dit, l'antériorité historique - ancestrale - d'un groupe ne suffit pas (ou plus) à définir sa singularité contemporaine, et cela est sans doute particulièrement lisible en ce qui conceme les descendants d'esclaves africains au Brésil. À cet égard Roger Bastide [1967] pensait pouvoir en distinguer deux types: les "communautés africaines" et les «communautés nègres». Les premières seraient la parfaite réorganisation des modèles africains sur le sol brésilien, alors que les secondes auraient subi une trop forte pression du milieu environnant pour pouvoir maintenir et exprimer la mémoire de leur origine; elles ont alors dû inventer de nouvelles formes de vie en société. Cependant le sociologue français reconnaissait lui-même qu'entre ces deux états de culture il n'y a pas de réelle distinction, car même les communautés les plus «africaines» n'ont pu se réactualiser au Brésil sans modifier des pans entiers de leur organisation sociale et/ou religieuse. Mais, dès lors, qu'est-ce qui relie encore l'ensemble des populations de l'Amazonie contemporaine aux origines - réelles ou imaginaires - auxquelles elles sont supposées appartenir? 
Cette question a obtenu une telle pertinence dans le contexte brésilien (tout comme en d'autres lieux tristement célèbres de la planète) à mesure que la référence identitaire -ethnique - sert d'arme politique. La nouvelle Constitution, adoptée par ce pays en 1988, reconnaît l'existence de deux «minorités» dont l'État prétend à la fois protéger l'intégrité culturelle et garantir la reconnaissance légale de leurs terres. Outre les ethnies amérindiennes (non-acculturées), la seconde «minorité» concernée par ces louables articles constitutionnels, n'est pas comme on aurait pu le croire les Noirs ni même les Caboclo, mais les descendants desquilombos (Remanescentes de Quilombos). Ainsi, pour bénéficier aujourd'hui d'un droit à l'existence sur l'espace brésilien il faut être le descendant des groupes qui, historiquement, expriment avant tout leur opposition (voire leur illégitimité) face au monde colonial... Le Noir des quilombos et l'Indien se retrouvent ici

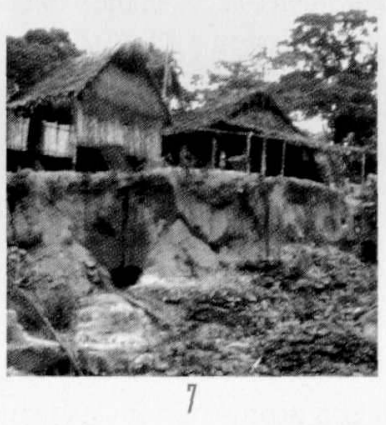

Terras de Preto) susceptibles d'exiger le statut de «Remanescente de Quilombos». Cette importante concentration rappelle l'étendue de l'économie esclavagiste, principalement orientée vers la production de coton et de canne à sucre, qui s'était développée dans la région jusqu'au milieu du XIXème siècle. À cette époque les deux-tiers de sa population était esclave, ce qui lui donnait largement le plus haut taux du Brésil ${ }^{[10]}$. Lorsque dans la seconde moitié du XIXème siècle le Maranhão connut une importante crise économique, l'ensemble du monde rural plongea dans l'immobilisme. De nombreuses fazendas furent abandonnées et leurs esclaves furent vendus aux exploitants miniers du Minas Gerais, en pleine expansion à cette époque. Mais ce vide économique facilita également la fuite d'esclaves et la multiplication des quilombos sur des espaces vierges ou abandonnés, bien que certains purent rapidement légaliser leur situation en s'affranétrangement associés dans leur lutte historique pour l'autonomie et l'intégrité. Outre le caractère troublant des critères constitutionnels, ce texte contribue aujourd'hui à cristalliser abusivement une référence «ethnique», comme s'il suffisait d'afficher son repentir face à cinq siècles de violences pour ériger une Nation démocratique. Ainsi, en deçà du problème anthropologique que soulève la référence ethnique en Amazonie, survient un problème politique et juridique qui ne va pas, on s'en doute, sans influencer le premier.

Rien que dans l'État brésilien du Maranhão on recense environ 400 communautés de Noirs (les chissant de leurs maîtres et en achetant quelquefois des terres laissées en friche. Cependant, depuis l'abolition définitive de l'esclavage, en 1888, la plupart de ces Terras de Preto ne sont toujours pas en possession d'un titre légal de propriété, tant et si bien que les conflits se multiplient avec de nouveaux grands propriétaires fonciers ayant racheté en masse les anciennes fazendas coloniales, et sur lesquelles ils ont «inopinément» découvert l'existence de villages peuplés de Noirs ou de Caboclo depuis parfois plusieurs siècles...

Au cœur même de l'Amazonie, le long du Rio Trombetas, il existe aussi une forte concen- 
tration de Remanescentes de Quilombos. Ici c'est la production de cacao qui monopolisa une importante quantité d'esclaves. Grâce à un relief fort accidenté qui ne permettait de se déplacer aisément que sur les plus importants cours d'eau, les esclaves marrons de Trombetas parvinrent à maintenir leurs quilombos en remontant les cascades de leur rivière à chaque fois que leurs mâtres tentaient d'envoyer des milices. Une étude sur les descendants actuels de ces quilombos [Acevedo \& Castro, 1993] a permis de mesurer avec quelle souplesse ils sont parvenus à s'adapter à leurs conditions particulières - tant au niveau du contexte sociologique que de l'environnement naturel- et à développer un système original d'existence. Les Noirs de Trombetas ont ainsi élaboré une économie mixte de type «agroextractiviste», alternant la pêche et l'agriculture domestique sur brûlis (principalement de manioc) durant l'été «sec»,

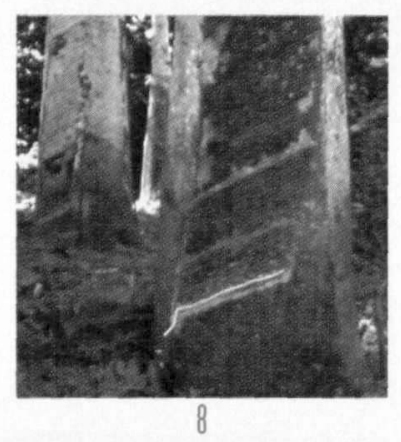

niant tous deux à leur manière la territorialité de ces Remanescentes de Quilombos.

Trombetas est un cas exemplaire, non seulement vis-à-vis de la singularité de son mode d'organisation sociale (qui, selon la typologie bastidienne, a dû innover face aux contraintes de son environnement naturel, social et culturel) mais surtout en fonction d'une problématique de l'ethnicité. La référence identitaire des Noirs de Trombetas, «construite dans leurs relations avec un territoire, (...) délimite à partir de dimensions objectives et subjectives, les contours reconnus d'un nous qui s'affirme face à la perception d'un autre, lui-même constitué par les groupes formant la société environnante, par les Blancs» [Acevedo \& Castro, 1993: 121]. Cette «identité ethnique» relève donc d'un parcours commun, non celui qui mena ces hommes sur les navires négriers, mais bien plus concrètement celui qui les opposa, depuis la création des avec la chasse et la récolte de produits forestiers (noix de Pará) en période de crue. Leur mode de vie, tout en s'adaptant au rythme des eaux (crues et décrues), renvoie à la fois à leur cheminement historique, leur rencontre avec l'Indien et leur intégration partielle à l'économie de marché. C'est à travers ce complexe ethno-sociologique que Rosa Acevedo \& Edna Castro [1993] défendent l'existence d'une autochtonie des Noirs de Trombetas face au double péril que leur imposent aujourd'hui, d'un côté, l'implantation d'une "Réserve Biologique» et, de l'autre, l'ouverture d'un important programme d'extraction minière, quilombos, à la société esclavagiste, ségrégationniste, puis démocratique. C'est donc ce rapport au quilombo qui constitue «l'ethnie» de Trombetas et non une vague - et même inexistante - référence à l'Afrique. Dès lors, même si cette spécificité culturelle est souvent niée, bafouée ou méprisée par la société environnante, elle n'en est pas moins une réalité ethnologique qui, pour compter aujourd'hui 6.000 individus, peut être rangée parmi les plus grands groupes «ethniques» de l'Amazonie.

Au rebours de cette composante endogène anthropologique- de l'ethnicité de ce type de com- 
munautés néo-amazoniennes, intervient aujourd'hui un contexte idéologique provoqué par l'étroitesse de la formulation juridique de la nouvelle Constitution brésilienne. L'application des Articles concernant les Remanescentes de Quilombos, loin de permettre une réflexion ou mieux une valorisation de ces «ethnies» néoamazoniennes (afro-indigènes ou «négro-caboclo»), s'égare plutôt dans des conflits de droit ou de preuves. Aujourd'hui, le moindre petit dépôt d'archives contenant des documents datant de la traite (un procès, un acte testamentaire, un rapport d'expédition policière, etc.), devient le lieu stratégique où les Afro-Brésiliens cherchent les armes juridiques leur permettant d'établir (ou de rétablir?) une continuité avec une communauté de fugitifs. Il ne s'agit donc pas de valoriser une singularité contemporaine, mais au contraire l'origine d'un conflit ségrégationniste. On tente moins alors de défendre la

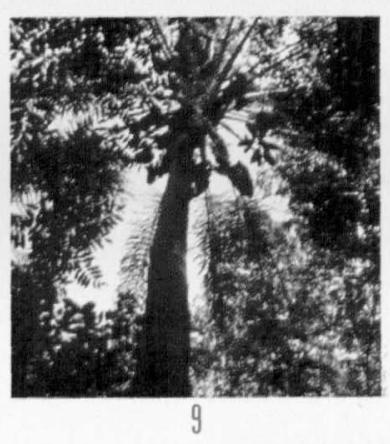

«descendants de quilombos» à qui l'État prétend offrir sa protection, qu'adviendra-t-il des trente millions restant, parmi lesquels se trouveront entre autres des «Noirs» sans quilombos, des «Indiens» sans réserves ou "acculturés», des «Caboclo» ou, tout simplement, des Hommes sans race ou sans ethrie?...

\section{CONCLUSION: \\ ségrégation raciale ou ségrégation ethnique?}

Au terme de ce rapide survol de l'espace multi-ethnique néo-amazonien, on ne peut que reposer la question sur ce qui relie les communautés de cette région aux origines auxquelles elles sont sensées appartenir? Nous avons pu constater qu'à chaque fois qu'il était question d'affirmer une référence ethnique celle-ci devait bien souvent faire face à une incontournable discontinuité historique. Cela ne signifie pas que les références au passé et à l'ancestralité aient disparu. Au contraire, celles-ci deviennent l'enjeu même de ces identités néo-amazoniennes; mais, simplement, l'histoire indigène de l'Amazonie s'est étendue à l'histoire coloniale du pays.

On constata même que, dans la majorité des cas, c'est davantage cette ethno-histoire coloniale qui focalise l'origine des groupes caboclo, noirs et même quelquefois amérindiens. La référence précoloniale (ou précolombienne pour les Indiens, ou encore pré-esclavagiste pour les Noirs), refoulée dans le mythe, a même quelquefois disparu ou s'est «atténuée» pour laisser place à une 
autochtonie davantage enracinée dans l'espace social et naturel devenu commun.

Si certains considèrent cette évolution avec mépris ou tristesse, montrant par-là leur attachement à ce qu'il y a de «sauvage» ou de "pré-historique" dans leur vision de l'autre ${ }^{[11]}$, là n'est sans doute pas le véritable danger que court l'Amazonie contemporaine. Mais cette attitude conduit bien souvent à une dérive idéologique qui s'apparente de manière inquiêtante à un racisme. l'etbricisation de l'Amazonie est sans doute un phénomène marquant aujourd'hui et prend, comme un peu partout dans le monde, la place qu'a eu la ségrégation raciale. Simplement, ce n'est plus avec des critères strictement physiques (ou génétiques) que les hommes s'opposent maintenant, mais avec des critères «ethniques", basés le plus souvent sur une référence exogène, c'est-à-dire extérieure à la pratique sociale et culturelle que l'on tente de singulariser, parce que figée dans une histoire culturelle méconnue. Que l'ethnie remplace la race, cela ne change rien. L'objectif est le même, qui consiste à justifier une discrimination ou un privilège; en l'occurrence ici, l'accès ou non à un espace vilal... Gageons que les intellectuels qui contribuent directement ou indirectement à orienter une politique en Amazonie - comme en tout autre espace social multi-ethnique - acceptent de substituer l'analyse et la réflexion à la tyrannie de leurs préjugés.

\section{REMERCIEMENTS}

I

Je remercie icj le Fonds des Bourses de l'Université Libre de Bruxelles pour m'avoir accordé le financement nécessaire à la réalisation de cet article. 


\section{NOTES}

\section{1}

Whitehead, 1993;

Curtenius Ronsevelt, 1993.

\section{2}

De nombreux ethnographes ont traité ce sujet. À titre indicatif Clastres, 1974.

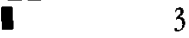

Pierre et Françoise Grenand evaluent la population de la Grande Amazonie à près de 700.000 Anérindiens pour plus de 30 millions de métis et de migrants. (In: Situation des populations indigenes des forêts denses bumides) (sous la dir de S. Bahuchet \& P. de Maret), Rapport rédigé pour la Communauté européenne (DCXI).

U.L.B./Lacito-CNRS, Juin 1993.

Cette démarche alla plus loin puisque, aux prises avec un travestisme identitaire, ils se sont choisi un nouvel ethnonyme, jugeant le terme "Tikuna» comme porteur d'un sens trop péjoratif [Pacheco de Oliveira, 1990].

\section{1} 5

Notamment, Parker,1985; Balée \& Posey, 1989; Nugent, 1993.

A cette fin on transporta, entre le XVIerrke et le XIX'une siècle, quelque onte millions d'esclaves à destination des Anériques: [Bastide, 1967].

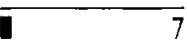

Pour lesquelles un travail d'ensemble reste encore à faire. Mentionnons cependant deux rélérences majeures les concemant: Ferretti, 1985, 1991: et Ferretti, 1991

\section{LÉGENDES DES PHOTOS}

\section{8}

Dans les cantiques chantés au moment des transes de possession donc au moment de leur apparition rituelle-ces Indiens se présentent comme ayant vécu sur la côte atlantique (dans les lagons de "Barra do (aririn) où ils seraient tous morts, sauf l'Indien Sapequara. Roi des Indiens ou Roi des Caboclo (Caboclo velho) selon les cantiques, qui dut alors fuir en Amazonie. Ces éléments rituels permettent à Mundicarmo Ferretti de proposer l'hypothèse qu'il s'agit ici des Indiens Cariri, effectivement massacrés en grand nombre par les Portugais au XVIItme siècle [Ferretti, M 1991, p.79 et 113].

9

Pour une analyse détaillée de cette tripartition cultuelle dans le culte Mina, Laveleye 1992, 1995.

\section{0}

D'après le recensement brésilien de 1819 reproduit par Ranos, 1943.

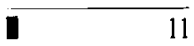

N'a-t-on pas dit ou écrit quelque part que l'archaïsme, ou le primitif, se trouve avant tout dans le regard que l'on porte sur l'autre...
1 : Culte afro-anérindien de "Tambor de Mina": une femme est possédée par un esprit de Caboclo de la forêt.

\{: Embarcation caboclo sur le Rio Preguiças, Maranhão (Brésil).

3. Quilombo de Frechal au Maranhão (Brésil) : les hommes de cette communauté négro-caboclo pêchent au filet.

4. Quilombo de Frechal au Maranhão (Brésil) : lies femmes de cette Terra de Preto pilent le manioc.

5: Essart caboclo sur le Rio Negro (Brésil) : les Caboclo fabriquent du charbon avec les arbres coupés.

6: Piste caboclo en forêt (Rio Solimões).

7: Habitation caboclo en Amazonie bolivienne.

8: Récolte du latex de l'hévéa par les Seringueiros de l'Amazonie.

9: Ansazonie. 